\title{
On the Configurational Statistics of Ethylene-Propylene Copolymers
}

\author{
J. E. MARK \\ The Department of Chemistry and the Macromolecular Research Center, The University of Michigan, Ann Arbor, Michigan 48104
}

(Received 5 May 1972)

\begin{abstract}
Mean-square unperturbed dimensions $\left\langle r^{2}\right\rangle_{0}$ and their temperature coefficient $d \ln \left\langle r^{2}\right\rangle_{0} / d T$ have been calculated for ethylene-propylene copolymers by means of the rotational isomeric state theory of chain molecules. Conformational energies required in the analysis are shown to be readily obtained from previous analyses of polyethylene and polypropylene, without additional approximations. Results thus calculated are reported as a function of chemical composition, chemical sequence distribution, and stereochemical composition of the propylene sequences. It is shown that $\left\langle r^{2}\right\rangle_{0}$ and $d \ln \left\langle r^{2}\right\rangle_{0} / d T$ for these copolymer chains should be most sensitive to chemical sequence distribution in the case where propylene sequences are highly isotactic in stereochemical structure. Calculations carried out using values of these variables thought to be appropriate for ethylene--propylene polymers experimentally investigated give values of $d \ln \left\langle r^{2}\right\rangle_{0} / d T$ which are in good agreement, in general, with those obtained from previously published force-temperature measurements on elongated networks of ethylene-propylene chains.
\end{abstract}

\section{INTRODUCTION}

A rotational isomeric state scheme $e^{1}$ has recently been developed in order to interpret dipole moments of vinyl copolymers and to characterize the general dependence of this configuration-dependent property on chain length, temperature, chemical sequence distribution, and stereochemical composition. The method was illustrated by application to the simplest case, vinyl copolymers in which the pendant groups have dipoles of different magnitude, but are of sufficiently similar chemical structure that the conformational energy of the chain is independent of chemical composition. The copolymers poly ( $p$-chlorostyrene, $p$-methylstyrene) and poly ( $p$-chlorostyrene, styrene) have this structural feature, and the conformational energies required in the calculation of their statistical properties had been reported previously by Williams and Flory. ${ }^{2}$ Dipole moments calculated for these two copolymers, using chemical sequence distributions characteristic of the reactivity ratios determined for these two sets of comonomers and random stereochemical sequence distributions, were found to be in good agreement with published experimental results ${ }^{3-5}$ obtained on a number of atactic samples of these copolymers in the bulk, nonglassy, amorphous state.

Since investigations of dipole moments are of course limited to polar molecules, it becomes of considerable importance to extend this earlier analysis to the calculation of the mean-square unperturbed dimensions $\left\langle r^{2}\right\rangle_{0}$ of nonpolar chemical copolymers. As in the case of the mean-square dipole moment $\left\langle\mu^{2}\right\rangle,{ }^{1}$ it is of interest to calculate this quantity as a function of chemical composition, chemical sequence distribution, and temperature. In the case of sequences of asymmetric units, such as vinyl sequences, the effect of stereochemical composition would also assume considerable importance..$^{6-9}$ The ideal systems for such investigation are the ethylene-propylene copolymers. From a theoretical point of view, their relatively simple structure is an attractive feature. From a practical point of view, these materials are one of the most important groups of commercial copolymers and their properties are therefore almost certain to be extensively studied in the future. In fact, ethylene-propylene copolymers crosslinked into network structures are, in general, stable rubberlike materials and some of their elastic properties have already been studied by a number of workers. ${ }^{10-12}$ The relevant fact here is that there is sufficient information on the force-temperature coefficient of elongated networks of these copolymers to estimate experimental values of the temperature coefficient $d \ln \left\langle r^{2}\right\rangle_{0} / d T$ of the unperturbed dimensions of these chains. This quantity is also accessible from the calculations to be carried out, thus providing at least a preliminary comparison of theory with experiment.

\section{THEORY}

In the study of chemical copolymers, the first requirement is the generation, or simulation, of chains having chemical and stereochemical structures which can be related to the nature of the comonomers and the conditions under which the copolymerization was carried out. As described previously, ${ }^{1}$ the distribution of monomer units along the chain is controlled by the conditional probabilities $p_{i j}$ that a chain ending in monomer type $i$ will add a unit of type $j$. In the case of binary copolymers, these probabilities are $p_{11}, p_{22}, p_{12}=1-p_{11}$, and $p_{21}=1-p_{22}$, and, according to copolymerization theory, ${ }^{13-16}$ are specified by

$$
\begin{aligned}
& p_{11}=r_{1} F_{1} /\left(r_{1} F_{1}+F_{2}\right), \\
& p_{22}=r_{2} F_{2} /\left(r_{2} F_{2}+F_{1}\right) .
\end{aligned}
$$

In these equations, $r_{1}$ and $r_{2}$ are reactivity ratios and are defined by

$$
\begin{aligned}
& r_{1}=k_{11} / k_{12}, \\
& r_{2}=k_{22} / k_{21},
\end{aligned}
$$

where $k_{i j}$ is the rate constant for the addition of monomer of type $j$ to a growing chain end of type $i$, and $F_{1}$ and $F_{2}=1-F_{1}$ are the mole fractions of monomers $M 1$ and $M 2$, respectively, in the monomer mixture 


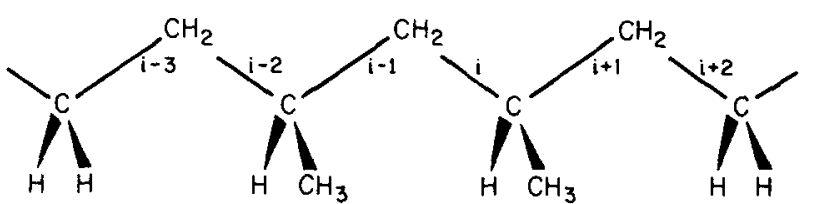

Fig. 1. The planar, all-trans conformation of a portion of an ethylene-propylene chain consisting of the chemical sequence ethylene, propylene, propylene, ethylene; the propylene sequence shown constitutes an isotactic $(d d)$ dyad. The lines of increasing and decreasing thickness represent bonds extending toward and away from the reader, respectively.

undergoing copolymerization. For very long chains prepared in a system of constant composition, the fraction $f_{1}$ of units of type 1 in the chain will, of course, be equal to the value of the probability $p_{1}$ that a monomer unit being incorporated into a growing chain is type 1 ; this quantity is given by ${ }^{15}$

$$
p_{1}=\left(r_{1} F_{1}^{2}+F_{1} F_{2}\right) /\left(r_{1} F_{1}^{2}+2 F_{1} F_{2}+r_{2} F_{2}^{2}\right) .
$$

The above set of equations indicates that specification of $f_{1}$ and the product $r_{1} r_{2}$ determines the values of $p_{11}$ and $p_{22}$ required to give a chain having the desired characteristics. Values of $p_{11}$ and $p_{22}$ thus calculated may be used in conjunction with a set of $x$ random numbers ranging in magnitude from 0 to 1 to simulate, by Monte Carlo methods, a copolymerization leading to a chain of $x$ monomer units. (The magnitude of the first number relative to $p_{1}$ is used to specify the chemical nature, or type, of the first unit in the chain; subsequent numbers are compared with either $p_{11}$ or $p_{22}$, depending on the nature of the preceding unit, to determine the type of monomer used in subsequent additions). The generation of the stereochemical structure of sequences of asymmetric units may also be carried out using the Monte Carlo technique on other sets of random numbers. [The locations and lengths of the sequences of asymmetric units are specified by the Monte Carlo generation of the chemical structure. For each such sequence, the magnitude of the first number generated is compared with 0.5 in order to specify the atomic configuration of the first unit; thereafter, occurrence of a number less than the chosen value of the replication probability $p_{r}$ is taken to specify an isotactic placement (replication), and a number greater than $p_{r}$, a syndiotactic placement (inversion).] For very long chains, the fraction $f_{r}$ of isotactic placements in the chain will be equal to the value chosen for $p_{r}$.

The rotational isomeric state model for vinyl chains has been extensively discussed elsewhere, ${ }^{6}$ as has its specific application to the calculation of the randomcoil, unperturbed dimensions $\left\langle r^{2}\right\rangle_{0}$ of both polyethylene chains, ${ }^{6,17}$ and polypropylene chains of various stereochemical compositions. ${ }^{6,18}$ Following the analyses of the two homopolymers, we adopt the rotational states trans $(l)$, gauche positive $\left(g^{+}\right)$, and gauche negative $\left(g^{-}\right)$ for the chain skeletal bonds, such as bonds $i-3$ through $i+2$ of Fig. 1. Since intramolecular steric interactions may be relatively large in ethylene-propylene chains, we allow for the displacement of these rotational states by an amount $\Delta \phi$ from their symmetric locations at 0 , 120 , and $-120^{\circ}{ }^{6}$ Specifically, these states are located at $-\Delta \phi, 120$, and $-120+\Delta \phi^{\circ}$, and $\Delta \phi, 120-\Delta \phi$, and $-120^{\circ}$ for two skeletal bonds leading into and out of a $\mathrm{C}^{\alpha}$ atom of $d$ configuration, respectively; the same two sets of rotational angles pertain to the two skeletal bonds leading, respectively, out of and into a $\mathrm{C}^{\alpha}$ atom of $l$ configuration. ${ }^{6,9,18}$ On the assumption of steric equivalence of $\mathrm{CH}, \mathrm{CH}_{2}$, and $\mathrm{CH}_{3}$ groups, a reasonable approximation already employed in the analysis of polypropylene chains, ${ }^{6,18}$ the same value of $\Delta \phi$ should characterize displacements of rotational states about $\mathrm{CH}_{2}-\mathrm{CH}_{2}$ bonds. These states are therefore located at $0,120-\Delta \phi$, and $-120+\Delta \phi^{0}{ }^{6,17}$

In the case of ethylene-propylene copolymers, structural similarities between the two comonomers make the characterization of intramolecular interactions and the assignment of statistical weight factors relatively. simple. Both repeat units consist of only $\mathrm{CH}, \mathrm{CH}_{2}$, and $\mathrm{CH}_{3}$ groups and, as will become obvious, there are no interactions occurring in ethylene-propylene chains not already characterized in previous studies of ethylene chains and propylene chains. The present analysis therefore closely follows the schemes employed for these two homopolymers. ${ }^{6,17,18} \mathrm{We}$ consider first those interactions dependent on only one bond rotational angle. Conformations in which a $\mathrm{CH}$ or $\mathrm{CH}_{2}$ group is syn to a $\mathrm{CH}_{2}$ group are assigned a statistical weight factor of $\sigma$, whereas those in which a $\mathrm{CH}$ group is syn to a $\mathrm{CH}_{3}$ group are given the factor $\tau$. Therefore, conformations in which $\mathrm{CH}$ is syn to both $\mathrm{CH}_{2}$ and $\mathrm{CH}_{3}$ are characterized by the product $\sigma \tau$. To facilitate comparison of the present analysis with that of polypropylene itself, we normalize the factors $\tau, \sigma, \sigma \tau$ to $\eta, 1, \tau$ where $\eta=\tau / \sigma .^{18}$ (In the approximation that $\mathrm{CH}, \mathrm{CH}_{2}$, and $\mathrm{CH}_{3}$ are sterically equivalent, $\eta=1.0$; it is however retained in the following scheme in order to permit determination of the sensitivity of the calculated results to this statistical weight factor.) Interactions dependent on two consecutive rotational angles, such as those about bonds $i-1$ and $i$ of Fig. 1, involve atoms or groups separated by four bonds. Except in the case where one of the interacting species is an $\mathrm{H}$ atom, all "pentane-type" interactions, which bring the participants into close proximity, are strongly repulsive. A single statistical weight factor $\omega \ll 1$ is therefore introduced to take into account the resulting near suppression of all conformations giving rise to such repulsions. Various combinations of these statistical weight factors may now be used to represent statistical weights for all possible conformations about consecutive bond pairs in ethylenepropylene chains.

For the purpose of calculating configuration-dependent properties, such statistical weights are used to construct statistical weight matrices $U_{i}$. In such matrices, rows are associated with rotational states about bond $i-1$ and columns, with states about bond $i$; both rows and columns are indexed in the order $t, g^{+}, g^{-}$. 
For $\mathrm{CH}_{2}-\mathrm{CH}_{2}-\mathrm{CH}_{2}$ (ethylene) bond pairs, the statistical weight matrix is designated $\mathrm{U}_{e}$ and has the form

$$
\mathrm{U}_{e}=\left[\begin{array}{ccc}
1 & \tau / \eta & \tau / \eta \\
1 & \tau / \eta & \tau \omega / \eta \\
1 & \tau \omega / \eta & \tau / \eta
\end{array}\right] .
$$

For a pair of bonds meeting at a $\mathrm{CHCH}_{3}$ group, the atomic configuration of the group must be specified. Adoption of the arbitrary $d, l$ convention (see Fig. 1) previously employed ${ }^{8,18}$ yields, for the statistical weight matrix characterizing bonds meeting at a $\mathrm{C}$ atom of $d$ configuration,

$$
\mathrm{U}_{d}=\left[\begin{array}{ccc}
\eta & 1 & \tau \\
\eta & 1 & \tau \omega \\
\eta & \omega & \tau
\end{array}\right] .
$$

By symmetry, the matrix $\mathrm{U}_{l}$ associated with a $\mathrm{C}$ atom of $l$ configuration is obtained by simple interchange ${ }^{6}$ of both the second and third rows and the second and third columns of $\mathrm{U}_{d}$. In the case of pairs of bonds separating two $\mathrm{CHCH}_{3}$ groups, the configuration of both groups must be specified. The statistical weight matrices for the dyads $d d$ and $d l$ are given by

$$
\begin{aligned}
\mathbf{U}_{d d} & =\left[\begin{array}{ccc}
\eta \omega & \tau \omega & 1 \\
\eta & \tau \omega & \omega \\
\eta \omega & \tau \omega^{2} & \omega
\end{array}\right], \\
\mathbf{U}_{d l} & =\left[\begin{array}{ccc}
\eta & \omega & \tau \omega \\
\eta \omega & 1 & \tau \omega \\
\eta \omega & \omega & \tau \omega^{2}
\end{array}\right] .
\end{aligned}
$$

The matrices $\mathrm{U}_{l l}$ and $\mathrm{U}_{l d}$ may be obtained from $\mathrm{U}_{d d}$ and $U_{d l}$, respectively, by the interchanges of rows and columns described above. Bond pairs $\mathrm{CHCH}_{3}-\mathrm{CH}_{2}-\mathrm{CH}_{2}$ are characterized by the matrices $\mathrm{U}_{d e}$ and $\mathrm{U}_{l e}$ where

$$
\mathrm{U}_{d e}=\left[\begin{array}{ccc}
\eta / \tau & \omega & 1 \\
\eta / \tau & 1 & \omega \\
\eta / \tau & \omega & \omega
\end{array}\right]
$$

and $\mathbf{U}_{l e}$ is related to $\mathrm{U}_{d e}$ by the symmetry operations already described. Similarly, the matrices $U_{e d}$ and $U_{e l}$ are associated with bond pairs $\mathrm{CH}_{2}-\mathrm{CH}_{2}-\mathrm{CHCH}_{3}$, with

$$
\mathrm{U}_{e d}=\left[\begin{array}{ccc}
\eta & \tau & 1 \\
\eta & \tau \omega & \omega \\
\eta \omega & \tau \omega & 1
\end{array}\right]
$$

and $\mathrm{U}_{e l}$ having the usual relationship to $\mathrm{U}_{e d}$.
Using the above statistical weight matrices, the configuration partition function $Z$ for an ethylenepropylene chain of $n$ bonds, or $x=n / 2$ repeat units, can be written ${ }^{6}$

$$
Z=\mathrm{J}^{*}\left[\prod_{i=2}^{n-1} \mathrm{U}_{i}\right] \mathrm{J},
$$

where $\mathrm{J}^{*}=[100]$ and $\mathrm{J}$ is the transpose of [111]. Calculation of mean-square unperturbed dimensions from skeletal bond vectors 1 requires construction of Cartesian coordinate systems about each bond in the chain backbone. If the $x$ axis is taken along bond $i$, and the positive $y$ axis is taken in the plane determined by bonds $i$ and $i-1$ so as to make an acute angle with bond $i-1$, then the matrix $\mathbf{T}_{i}$ which transforms a vector in coordinate system $i+1$ into that of system $i$ is ${ }^{6}$

$$
\mathrm{T}_{i}=\left[\begin{array}{ccc}
\cos \theta_{i} & \sin \theta_{i} & 0 \\
\sin \theta_{i} \cos \phi_{i} & -\cos \theta_{i} \cos \phi_{i} & \sin \phi_{i} \\
\sin \theta_{i} \sin \phi_{i} & -\cos \theta_{i} \sin \phi_{i} & -\cos \phi_{i}
\end{array}\right],
$$

where $\theta$ is the bond angle supplement. The rotational angle $\phi$ is defined relative to a value of zero for the trans state, with positive values corresponding to righthanded rotations, as described previously. ${ }^{6,8}$ The characteristic ratio is then given by ${ }^{6}$

$$
\left\langle r^{2}\right\rangle_{0} / n l^{2}=2\left(Z n l^{2}\right)^{-1} I^{*} \mathrm{G}_{1}\left[\prod_{i=2}^{n-1} \mathrm{G}_{i}\right] \mathrm{G}_{n} \mathrm{I},
$$

where $I^{*}$ is the row vector consisting of a single unity followed by 14 zeros, and $I$ is the column vector consisting of 12 zeros followed by three unities. The generator matrix $\mathbf{G}$ has the form ${ }^{6}$

$$
\mathbf{G}_{i}=\left[\begin{array}{ccc}
\mathbf{U} & \left(\mathbf{U} \otimes \mathbf{1}^{T}\right)\|\mathbf{T}\| & \left(l^{2} / 2\right) \mathrm{U} \\
\mathbf{0} & \left(\mathrm{U} \otimes E_{3}\right)\|\mathbf{T}\| & \mathrm{U} \otimes \mathbf{1} \\
0 & 0 & \mathrm{U}
\end{array}\right]_{i},
$$

where the subscript on the brackets refers to all quantities contained therein. The symbol $\otimes$ designates the matrix direct product, ${ }^{6}$ and $\mathbf{l}^{T}$ and $l$ are the transpose and magnitude, respectively, of the skeletal bond vector. The matrices $\mathbf{G}_{1}$ and $\mathbf{G}_{n}$ are atypical since they characterize the first and last bonds, respectively, of the chain. The statistical weight matrix in these two generator matrices is simply the unit matrix $\mathbf{E}_{3}$ of order three. The pseudodiagonal matrices $\left\|\mathbf{T}_{i}\right\|$ are defined by

$$
\left\|\mathbf{T}_{i}\right\|=\left[\begin{array}{lll}
\mathbf{T}_{i}\left(\phi_{t}\right) & & \\
& \mathbf{T}_{i}\left(\phi_{o}{ }^{+}\right) & \\
& & \mathbf{T}_{i}\left(\phi_{\sigma^{-}}\right)
\end{array}\right] .
$$

As described above, $\mathbf{U}_{i}$ and $\mathbf{G}_{i}$ depend in general on the chemical and stereochemical nature of both the monomer unit in which bond $i$ is located and its predecessor 
TABLE I. Average chemical sequence lengths in copolymers of degree of polymerization $x=100$.

\begin{tabular}{|c|c|c|c|c|c|c|c|c|c|c|}
\hline \multirow[b]{2}{*}{$r_{1} r_{2}$} & \multicolumn{2}{|c|}{$p_{2}=0.1$} & \multicolumn{2}{|c|}{0.2} & \multicolumn{2}{|c|}{0.3} & \multicolumn{2}{|c|}{0.4} & \multicolumn{2}{|c|}{0.5} \\
\hline & $n_{\mathrm{L}}$ & $n_{2}$ & $n_{1}$ & $n_{2}$ & $n_{1}$ & $n_{2}$ & $n_{1}$ & $n_{2}$ & $n_{1}$ & $n_{2}$ \\
\hline 0.00 & $\ldots$ & ... & $\ldots$ & $\ldots$ & $\ldots$ & $\ldots$ & ... & $\ldots$ & 1.00 & 1.00 \\
\hline 0.01 & 11.50 & 1.04 & 4.78 & 1.00 & 2.45 & 1.01 & 1.59 & 1.02 & 1.08 & 1.15 \\
\hline 0.10 & 11.47 & 1.06 & 4.98 & 1.05 & 2.52 & 1.11 & 1.79 & 1.21 & 1.35 & 1.34 \\
\hline 1.00 & 12.70 & 1.12 & 5.63 & 1.25 & 3.62 & 1.48 & 2.59 & 1.71 & 2.10 & 2.03 \\
\hline 10.0 & 24.28 & 2.93 & 11.69 & 3.69 & 8.68 & 4.07 & 5.32 & 4.02 & 4.59 & 4.30 \\
\hline 100.0 & 49.57 & 5.60 & 21.31 & 6.65 & 14.78 & 6.67 & 11.56 & 8.90 & 7.84 & 8.73 \\
\hline
\end{tabular}

along the chain. The precise sequences of U's and G's appearing in Eqs. (12) and (14) are specified by Monte Carlo methods, as already described here and elsewhere. ${ }^{1}$

\section{NUMERICAL RESULTS AND DISCUSSION}

Average chemical sequence lengths $n_{1}$ and $n_{2}$ of comonomers 1 and 2 in chains of degree of polymerization $x=100$ were obtained by Monte Carlo methods as described in the preceding section. Sequences were accepted as being representative only if the differences between $f_{1}$ and $p_{1}$ and between $f_{r}$ and $p_{r}$ were both less than 0.05 ; other sequences were discarded. Results obtained are given as a function of $p_{2}$, for values of $r_{1} r_{2}$ ranging from 0.0 to 100.0 , in Table I; each entry is the average calculated from five chains meeting the criteria cited above. Values of $n_{1}$ and $n_{2}$ for $p_{2}>0.5$ can of course be obtained by simple interchange of the subscripts 1 and 2. Monte Carlo generation of stereochemical sequences were carried out for $p_{r}=0.95,0.50$, and 0.05 , corresponding to propylene sequences which would be highly isotactic, atactic, and highly syndiotactic, respectively. Isotactic sequence lengths vary of course somewhat with the length of the propylene sequence being generated. In the limiting case of 100 units, these calculations led, also in the case of five successful trials, to average isotactic sequence lengths of $16.38,1.974$, and 1.067 for $p_{r}=0.95,0.50$, and 0.05 , respectively.

Calculations of $\left\langle r^{2}\right\rangle_{0} / n l^{2}$ were carried out using (i) $r_{1} r_{2}=0.01,1.0,10.0$, and 100.0, (ii) $p_{r}=0.95,0.50$, and 0.05 , (iii) bound lengths of $1.53 \AA$ and bond angles of $112^{\circ}$ for all skeletal bonds, ${ }^{6,17,18}$ (iv) $\Delta \phi=0$ and $10^{\circ},{ }^{17,18}$ and (v) statistical weight factors appropriate for temperatures of $-25,25$, and $75^{\circ} \mathrm{C}$. On the basis of the previous analyses of polyethylene and polypropylene, ${ }^{17,18}$ the values adopted for these statistical weight factors at $25^{\circ} \mathrm{C}$ were $\tau=0.43$ and $\omega=0.034$; for reasons already presented, the factor $\eta$ was set equal to unity. Values of these quantities at the other temperatures cited were calculated on the assumption that each factor could be expressed as a Boltzmann factor in the conformational energy. Since experimental results are almost invariably obtained on polymers of high molecular weight, all calculations were carried out using a degree of polymerization $x$ of 100 , a value sufficiently high in general that $\left\langle r^{2}\right\rangle_{0} / n l^{2}$ is very close to its value in the limit of very large $x$. [It is only in the case of very large values of the characteristic ratio, e.g., those for very extended chains such as nearly perfect isotactic poly ( $\alpha$-olefins), that significantly larger values of $x$ would be required for $\left\langle r^{2}\right\rangle_{0} / n l^{2}$ to become essentially independent of $x$.]

Calculated values of the characteristic ratio at $25^{\circ} \mathrm{C}$ for ethylene-propylene copolymers having $p_{r}=0.95$ (high isotacticity) are shown in Fig. 2; the copolymer composition variable $p_{2}=1-p_{1}$ is the probability of occurrence of propylene units. Each point shown represents the average of the results obtained on five Monte Carlo chains; in this and the following figures, lines of length twice the standard deviation in the set of results have been drawn through the points in those cases in which this length exceeded the diameter of the circles used to locate the points. (For purposes of clarity only the points used to locate the curves corresponding to

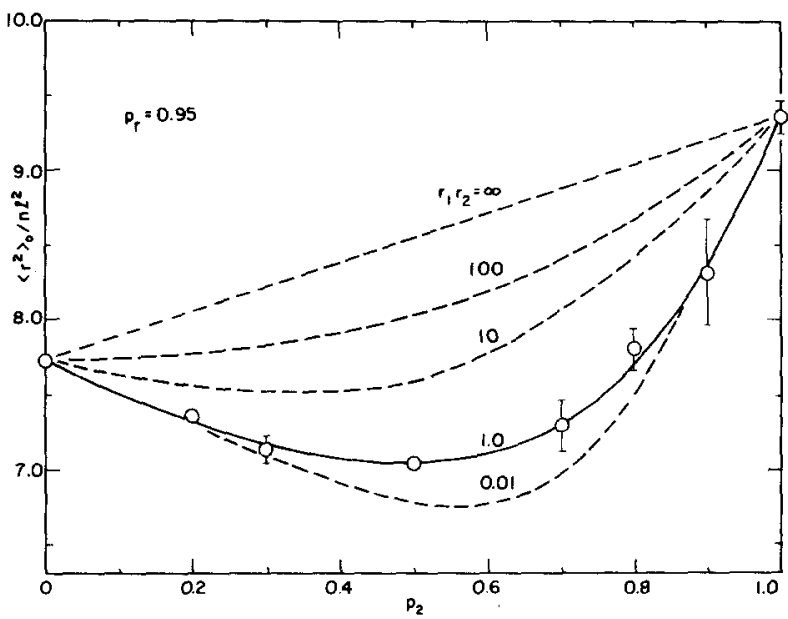

FIG. 2. The effect of chemical composition on the characteristic ratio, at $25^{\circ}$, of ethylene-propylene copolymers having propylene sequences which are highly isotactic in stereochemical structure. In Figs. $2-4$, results pertain to chains having $x=100$ units, with rotational states located at $\phi=0, \pm 120^{\circ}$ (displacement $\Delta \phi=0^{\circ}$ ). The chemical composition of the chains is characterized by the probability $p_{2}$ of occurence of propylene units, the chemical sequence distribution by the reactivity ratio product $r_{1} r_{2}$, and the stereochemical composition by the replication probability $p_{r}$ (probability of isotactic placement). Each circle shown locates the average result calculated for five Monte Carlo chains, and the lines through these points represent standard deviations (see text). For purposes of clarity, calculated points and standard deviation bars are shown only for the case $r_{1} r_{2}=1.0$. 
$r_{1} r_{2}=1.0$ are shown in Figs. 2-4). As shown in Table I, results calculated for increasing $r_{1} r_{2}$ at constant $p_{2}$ correspond to an increasing tendency to form long sequences or "blocks." The case $r_{1} r_{2}=\infty$ yields a mixture of homopolymers in which the mole fraction of chains composed entirely of units of type 2 is simply $p_{2}$; this is the origin of the linear dependence of $\left\langle r^{2}\right\rangle_{0} / n l^{2}$ on $p_{2}$ shown for this case in Figs. 2-4. The most striking feature of the results for $p_{r}=0.95$ is the predicted marked decrease in the characteristic ratio of either homopolymer upon the addition of units of the other type, when such units are widely dispersed (short sequence lengths $n_{1}$ and $n_{2}$ ). In the case of copolymer chains that contain predominantly ethylene, the propylene units cause departures from the extended all-trans conformations, which are the low energy forms for ethylene sequences; this results, of course, from the fact that there are two conformations ( $t$ and either $g^{+}$ or $g^{-}$) which are of essentially equal probability in the case of propylene units. ${ }^{6,18}$ For chains consisting mostly of (highly isotactic) propylene, the ethylene units disrupt the extended $3_{1}$ helical sequences, ${ }^{6,18}$ thus again causing a decrease in the characteristic ratio. As would be expected from the above argument, this effect decreases as the average sequence lengths increase (increase in $r_{1} r_{2}$ ). For large values of $p_{r}$, it seems that at least under some conditions measurement of the characteristic ratio might serve as a means of determining values of $r_{1} r_{2}$, and thus average sequence lengths, in ethylene-propylene copolymers. A similar conclusion was reached in the case of the mean-square dipole moments of poly ( $p$-chlorostyrene, $p$-methylstyrene) and poly( $p$-chlorostyrene, styrene) copolymers, but in the case of significantly syndiotactic chains $\left(p_{r}<0.50\right)$.

Figure 3 shows the results obtained at $25^{\circ} \mathrm{C}$ for the case $p_{r}=0.50$ (atacticity). Addition of propylene units to a chain which is predominantly ethylene should cause a significant decrease in the characteristic ratio, for reasons already cited. On the other hand, however, addition of ethylene units to a chain which is predominantly atactic propylene has little effect on $\left\langle r^{2}\right\rangle_{0} / n l^{2}$

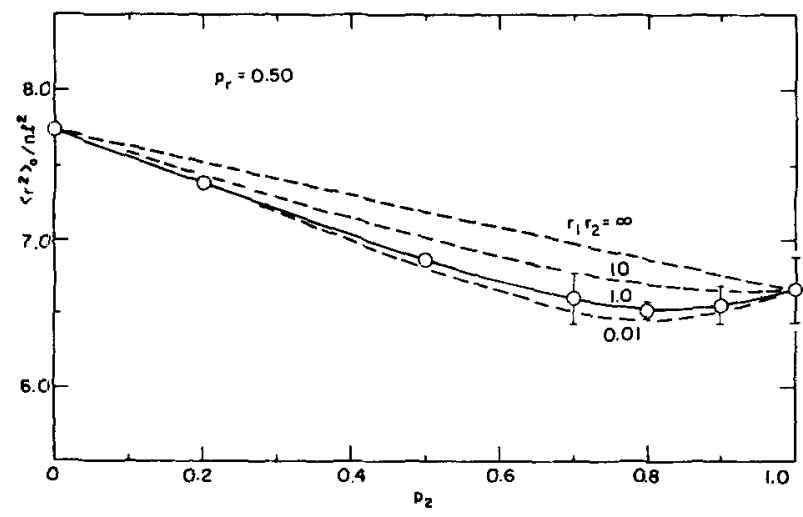

Fig. 3. The characteristic ratio at $25^{\circ}$ of ethylene-propylene copolymers having atactic propylene sequences; see caption for Fig. 2.

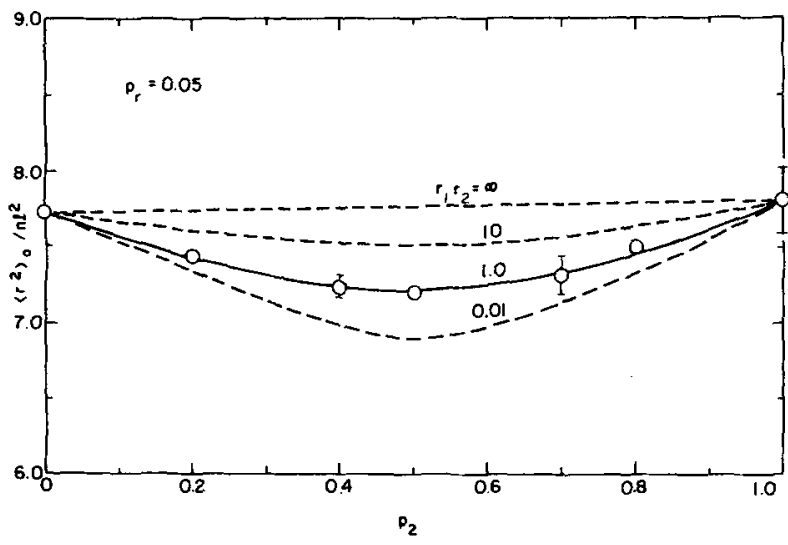

FIG. 4, The characteristic ratio at $25^{\circ}$ of ethylene-propylene copolymers having highly syndiotactic propylene sequences; see caption for Fig. 2 .

since the propylene sequences, being essentially random in stereochemical structure are not present in any regular, extended conformation. This stereochemical and conformational randomness also accounts for the lack of a strong dependence of the characteristic ratio on $r_{1} r_{2}$ at constant $p_{2}$. The results obtained at $25^{\circ} \mathrm{C}$ for $p_{r}=0.05$ (high syndiotacticity) are shown in Fig. 4. This case is in a sense intermediate to the cases $p_{r}=0.95$ and 0.50 since there is a stereochemical and conformational regularity in the syndiotactic propylene sequences in such chains, but this conformational regularity and associated chain extension are not as pronounced as they are in highly isotactic chains. ${ }^{6,18}$

The effect of temperature on the dependence of $\left\langle r^{2}\right\rangle_{0} / n l^{2}$ on $r_{1} r_{2}$ was investigated for the case $p_{r}=0.95$, by adjustment of the statistical weight, Boltzmann factors to -25 and $75^{\circ} \mathrm{C}$. Results for $p_{2}=0.5$ (the only possible value for the limiting case $r_{1} r_{2}=0.00$ ) are shown in Fig. 5 where open, left-filled, and right-filled circles refer to $25,-25$, and $75^{\circ} \mathrm{C}$, respectively. Decrease in temperature enhances the sensitivity of $\left\langle r^{2}\right\rangle_{0} / n l^{2}$ to $r_{1} r_{2}$ because of the increase in the prevalence of low energy, highly extended $3_{1}$ helical conformations in the isotactic propylene sequences. ${ }^{6,18}$ The dotted curve in this figure shows the effect of altering the locations of rotational states by increase in $\Delta \phi$ from 0 to $10^{\circ}$, at $25^{\circ} \mathrm{C}$. The change observed in $\left\langle r^{2}\right\rangle_{0} / n l^{2}$ is relatively small, due to the existence of two partially compensating effects. Increase in $\Delta \phi$ tends to decrease the characteristic ratio in the case of isotactic propylene sequences because this alteration decreases the degree of extension of the helical conformations. ${ }^{6,18}$ On the other hand, increase in $\Delta \phi$ increases this ratio for ethylene sequences since in this case ${ }^{6,17}$ gauche states are revised to locations closer to trans states, which are conformations of higher extension.

The dependence of the characteristic ratio on the statistical weight factors was calculated for ethylenepropylene chains at values of these factors appropriate to $50^{\circ} \mathrm{C}$, a temperature convenient for later comparison of theory with experiment. For purposes of economy 
TABLE II. The effects of the statistical weight factors and temperature on the mean-square unperturbed dimensions of ethylene-propylene copolymers. ${ }^{n}$

\begin{tabular}{|c|c|c|c|c|c|c|c|}
\hline$p_{r}$ & $\Delta \phi(\mathrm{deg})$ & $r_{1} r_{2}$ & $p_{2}$ & $\partial \ln \left\langle r^{2}\right\rangle_{0} / \partial \ln \eta$ & $\partial \ln \left\langle r^{2}\right\rangle_{0} / \partial \ln \tau$ & $\partial \ln \left\langle r^{2}\right\rangle_{0} / \partial \ln \omega$ & $-10^{3} d \ln \left\langle r^{2}\right\rangle_{0} / d T$ \\
\hline \multirow[t]{8}{*}{0.95} & 0 & 1.0 & 0.0 & 0.46 & -0.43 & -0.018 & 1.2 \\
\hline & & & 0.2 & 0.46 & -0.37 & -0.027 & 1.2 \\
\hline & & & 0.5 & 0.34 & -0.29 & -0.080 & 1.5 \\
\hline & & & 0.8 & 0.20 & -0.20 & -0.21 & 2.5 \\
\hline & & & 1.0 & 0.05 & -0.15 & -0.38 & 4.0 \\
\hline & 0 & 0.0 & 0.5 & 0.50 & -0.25 & -0.054 & 1.1 \\
\hline & 0 & 100.0 & 0.5 & 0.33 & -0.33 & -0.10 & 1.8 \\
\hline & 10 & 1.0 & 0.5 & 0.31 & -0.23 & -0.057 & 1.1 \\
\hline \multirow[t]{6}{*}{0.50} & 0 & 1.0 & 0.2 & 0.46 & -0.38 & -0.029 & 1.2 \\
\hline & & & 0.5 & 0.45 & -0.25 & -0.047 & 1.1 \\
\hline & & & 0.8 & 0.46 & -0.12 & -0.065 & 0.91 \\
\hline & & & 1.0 & 0.41 & -0.054 & -0.11 & 1.2 \\
\hline & 0 & 100.0 & 0.5 & 0.44 & -0.28 & -0.047 & 1.2 \\
\hline & 10 & 1.0 & 0.5 & 0.39 & -0.21 & -0.036 & 0.89 \\
\hline \multirow[t]{6}{*}{0.05} & 0 & 1.0 & 0.2 & 0.47 & -0.39 & -0.030 & 1.2 \\
\hline & & & 0.5 & 0.54 & -0.25 & -0.054 & 1.1 \\
\hline & & & 0.8 & 0.56 & -0.15 & -0.080 & 1.1 \\
\hline & & & 1.0 & 0.52 & -0.083 & -0.12 & 1.4 \\
\hline & 0 & 100.0 & 0.5 & 0.51 & -0.28 & -0.058 & 1.2 \\
\hline & 10 & 1.0 & 0.5 & 0.46 & -0.24 & -0.056 & 1.1 \\
\hline
\end{tabular}

a Calculated for a degree of polymerization of 100 ; coefficients have been evaluated at values of the statistical weight factors pertaining to $50^{\circ} \mathrm{C}$.

these calculations were carried out on the single Monte Carlo chain judged on the basis of the previous calculations to be most representative of chains of each of the specified values of $p_{2}, r_{1} r_{2}$, and $p_{r}$. The results are given in Columns 5-7 of Table II. Temperature coefficients of $\left\langle r^{2}\right\rangle_{0}$, calculated from these results in the usual manner, ${ }^{19}$ assuming the statistical weight factors to be Boltzmann factors, ${ }^{6}$ are given in the final column of the table. Only one set of results is presented for $r_{1} r_{2}=0.0$; this assignment yields sequence lengths of unity, thus making use of the quantity $p_{r}$ unnecessary. Similarly, there is only one entry for $p_{2}=0.0$ (ethylene homopolymer).

The temperature coefficient of $\left\langle r^{2}\right\rangle_{0}$ is seen to be invariably negative, with values of $-10^{3} d \ln \left\langle r^{2}\right\rangle_{0} / d T$ being in the range 0.9-1.5 except for chains consisting predominantly of very long, highly isotactic propylene sequences. As can be seen from the table, the temperature coefficient in the case of chains made up primarily of ethylene units is due largely to transitions from trans to gauche states, characterized by the factor $\tau$, as the temperature is increased..$^{6,17}$ On the other hand, the factor $\omega$ plays a more important role in the case of chains consisting predominantly of propylene units. (In general, the characteristic ratio does depend significantly on $\eta$, as is shown in Table II; the temperature coefficient of $\left\langle r^{2}\right\rangle_{0}$, however, receives no contribution from $\eta$ in the approximation $\eta=1.0$.) The nature of the dependence of the temperature coefficient on $p_{2}$ depends on the stereochemical structure of the propylene sequences since the relative magnitude of $\left\langle r^{2}\right\rangle_{0} / n l^{2}$ for propylene chains or sequences is strongly dependent on $p_{t^{*}}{ }^{6,18}$ The results shown also predict that the temperature coefficient should depend significantly on $r_{1} r_{2}$ at $p_{2}=0.5$ only for relatively large values of $p_{r}$. Finally, alteration of $\Delta \phi$ from 0 to $10^{\circ}$ has only a modest effect on the temperature coefficient in the case of large $p_{r}$, and essentially a negligible effect otherwise.

\section{COMPARISON OF THEORY WITH EXPERIMENT}

At the present time, unfortunately, there do not appear to be any published experimental studies from which values of $\left\langle r^{2}\right\rangle_{0} / n l^{2}$ itself may be calculated. There are, however, several investigations ${ }^{10-12}$ of the forcetemperature dependence of elongated, noncrystalline networks prepared from these copolymers. As is now well known, such information may be used to estimate values of the temperature coefficient of the unperturbed dimensions of the chains making up the network. ${ }^{20}$

The comparison of theoretical with experimental values or this coefficient is complicated by the fact that there appears to be considerable uncertainty and disagreement regarding the reactivity ratios of the ethylene-propylene comonomer pair, and the composition and structure of a "typical" ethylene-propylene copolymer (i.e., one prepared in the vicinity of room temperature with a Ziegler-Natta catalyst system). Early determinations of reactivity ratios for such 
typical copolymerizations in the range $0-75^{\circ} \mathrm{C}$ gave $r_{1} r_{2}=1.1 \pm 0.2 .^{21}$ Cozewith and Ver Strate, ${ }^{22}$ however, have recently concluded that these results are unreliable because of difficulties ${ }^{23}$ in the usual methods of chemical analysis of these copolymers and, at least in the case of homogeneous Ziegler-Natta catalysts, the existence of previously unrecognized complexities in the polymerization mechanism. These workers suggest the range $r_{1} r_{2}=0.1-0.5$ for ethylene-propylene copolymers prepared using homogeneous Ziegler-Natta catalysts in the vicinity of room temperature. An additional uncertainty results from the fact that either heterogeneous or homogeneous catalysts are used to prepare ethylenepropylene copolymers; the former catalyst systems are thought to give propylene sequences of high isotacticity, but the latter apparently give propylene sequences significantly atactic in stereochemical structure. ${ }^{24}$ These complications may be particularly troublesome in the case of several relevant studies which have been carried out on materials prepared under inadequately specified conditions. Fortunately, as shown in Table II, the coefficient $d \ln \left\langle r^{2}\right\rangle_{0} / d T$ is not in general excessively dependent on the quantities in which there is uncertainty, thus permitting at least a preliminary comparison of theory with experiment.

In the first experimental study of relevance here, Barrie and Standen ${ }^{10}$ report force-temperature data for networks prepared from an ethylene-propylene copolymer, over the range $25-70^{\circ} \mathrm{C}$. The copolymer employed, "Enjay 404," has a value of $p_{2}$ of approximately $0.5,{ }^{25}$ presumably short sequences $\left(r_{1} r_{2} \leq 1.0\right)$, but unspecified stereochemical structure. This study yields the result $-10^{3} d \ln \left\langle r^{2}\right\rangle_{0} / d T=1.6( \pm 0.1)$, in good agreement with the range 1.1-1.5 shown in Table II for the specified value of $p_{2}$, the indicated range of $r_{1} r_{2}$, and $p_{r}=0.95$. (Even if $p_{r}$ is revised to a value as low as 0.50 , there is still fair agreement between theoretical

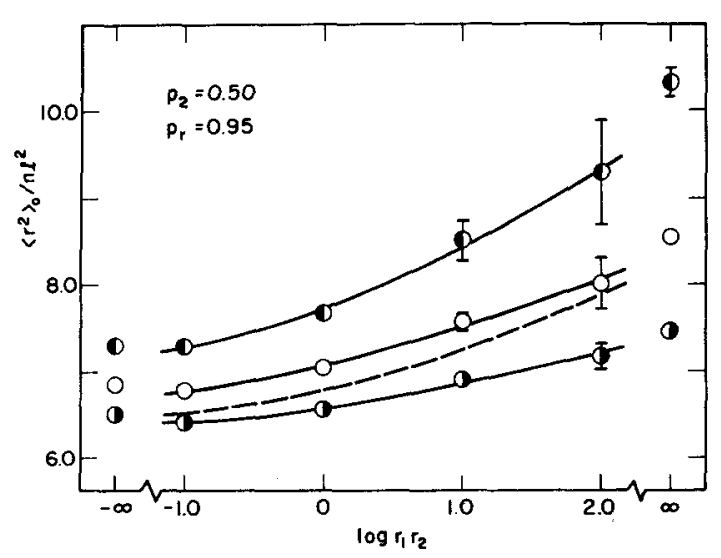

Fig. 5. The dependence of the characteristic ratio on $\log r_{1} r_{2}$ for ethylene-propylene copolymers having $x=100$ units equimolar chemical composition and highly isotactic propylene sequences. All circles locate results calculated for $\Delta \phi=0^{\circ}$; unfilled circles refer to $25^{\circ} \mathrm{C}$, left-filled circles to $-25^{\circ} \mathrm{C}$, and right-filled circles to $75^{\circ} \mathrm{C}$. The dotted curve shows results obtained for $\Delta \phi=10^{\circ}$, at $25^{\circ} \mathrm{C}$. and experimental values). Flisi, Crespi, and Valvassori ${ }^{12}$ have carried out a similar study, from -50 to $70^{\circ} \mathrm{C}$, on ethylene-propylene copolymers prepared, unfortunately, under entirely unspecified conditions. They obtain values of $-10^{3} d \ln \left\langle r^{2}\right\rangle_{0} / d T$ of $1.4( \pm 0.6)$ and $1.6( \pm 0.4)$, at approximately $50^{\circ} \mathrm{C}$, for the compositions $p_{2}=0.32$ and 0.49 , respectively. These results are in good agreement with theory and would seem to give preliminary confirmation of the predicted increase in the temperature coefficient with increase in propylene content, but the large uncertainties in the experimental results make this latter conclusion extremely tentative. In the final study using this approach, Opschoor and Prins ${ }^{11}$ report force-temperature data, from 20 to $70^{\circ} \mathrm{C}$, on networks of ethylene-propylene copolymers thought to be very nearly alternating $\left(n_{1}=\right.$ $n_{2}=1.0$ ) by virtue of their preparation by the almost complete $(\geq 92 \%)$ hydrogenation of poly (cis-1,4-isoprene). The result from this study, $-10^{3} d \ln \left\langle r^{2}\right\rangle_{0} / d T=$ $0.1 \pm(0.2)$ in the vicinity of $50^{\circ} \mathrm{C}$, is in poor agreement with the calculated result, 1.1 , obtained for $r_{1} r_{2}=0.0$. If this experimental result is not vitiated by the presence of significant amounts of unhydrogenated units along the chain, it is perhaps at least somewhat relevant that theory does predict that a perfectly alternating ethylene-propylene copolymer should have the smallest value of the temperature coefficient of the unperturbed dimensions of any ethylene-propylene copolymer of equimolar chemical composition. Finally, it should be mentioned that Cirlin, Gebhard, and Shen $^{26}$ have recently reported a value of the temperature coefficient of the shear modulus of an ethylene-propylene copolymer containing $48 \%$ propylene. They obtain a value of -0.1 for $-10^{3} d \ln \left\langle r^{2}\right\rangle_{0} / d T$, in poor agreement with both theory and other experimental results already cited..$^{10,12}$

\section{CONCLUSIONS}

The preceding analysis should be of considerable interest in the characterization of chemical copolymers since ethylene-propylene copolymers constitute one of only a relatively few cases which can be reliably treated using structural information and conformational energies derived exclusively from the corresponding homopolymers. Comparison of theoretical with experimental values of the temperature coefficient of the unperturbed dimensions, although preliminary, gives considerable support to the model proposed. Experimental studies of the unperturbed dimensions themselves, however, particularly as a function of chemical and stereochemical composition and sequence distribution, would obviously be highly desirable for further comparison of theory with experiment.

\section{ACKNOWLEDGMENT}

The author acknowledges, with gratitude, the financial support provided for this research by the National Science Foundation through Grant GP-16028. 
1 J. E. Mark, Polymer Preprints 13 (1), 135 (1972).

2 A. D. Williams and P. J. Flory, J. Am. Chem. Soc, 91, 3111 (1969).

${ }^{3}$ F. H. Smith, L. C. Corrado, and R. N. Work, Polymer Preprints 12 (1), 64 (1971).

${ }_{4}^{4}$ R. N. Work and Y. M. Tréhu, J. Appl. Phys. 27, 1003 (1956).

5 T. M. Birshtein, L. L. Burshtein, and O. B. Ptitsyn, Zh. Tech.

Fiz. 7, 896 (1959) [Sov. Phys. Tech. Phys. 4, 810 (1959)].

${ }^{6}$ P. J. Flory, Statistical Mechanics of Chain Molecules (Interscience, New York, 1969).

${ }^{7}$ Reference 8 and articles on vinyl polymers cited therein.

8 J. E. Mark, J. Chem. Phys. 56, 451 (1972).

' J. E. Mark, J. Chem. Phys. 56, 458 (1972).

$10 \mathrm{~J}$. A. Barrie and J. Standen, Polymer 8, 97 (1967).

11 A. Opschoor and W. Prins, J. Polymer Sci. Pt. C 16, 1095 (1967).

${ }_{12}$ U. Flisi, G. Crespi, and A. Valvassori, Kautschuk Gummi 22, 154 (1969); reprinted in Rubber Chem. Technol. 43, 778 (1970).

${ }_{13}$ See, for example, Refs. 14-16.

${ }^{14}$ T. Alfrey, Jr., J. J. Bohrer, and H. Mark, Copolymerization (Interscience, New York, 1952).

${ }_{15}$ P. J. Flory, Principles of Polymer Chemistry (Cornell U. P., Ithaca, N. Y., 1953), Chap. 5.
${ }^{16}$ G. E. Ham, in Copolymerization, edited by G. E. Ham (Interscience, New York, 1964).

${ }^{17}$ A. Abe, R. L. Jernigen, and P. J. Flory, J. Am. Chem. Soc. 88, 631 (1966).

${ }_{18}$ P. J. Flory, J. E. Mark, and A. Abe, J. Am. Chem. Soc. 88, 639 (1966)

19 P. J. Flory, V. Crescenzi, and J. E. Mark, J. Am. Chem. Soc. 86, 146 (1964).

${ }^{20}$ See, for example, A. Ciferri, C. A. J. Hoeve, and P. J. Flory, J. Am. Chem. Soc. 83, 1015 (1961).

${ }^{21}$ For a compilation, see H. F. Mark, B. Immergut, E. H. Immergut, L. J. Young, and K. I. Beynon, in Polymer Handbook, edited by J. Brandrup and E. H. Immergut (Interscience, New York, 1966).

${ }_{22} \mathrm{C}$. Cozewith and G. Ver Strate, Macromolecules 4, 482 (1971).

${ }^{23}$ I. J. Gardner, C. Cozewith, and G. Ver Strate, Rubber Chem. Technol. 44, 1015 (1971).

${ }_{24}^{4}$ See, for example, J. Boor, Jr, Macromol. Rev. 2, 115 (1967).

${ }^{25}$ Some limited information on the material studied was provided by the manufacturer, the Enjay Chemical Co. (private communication).

${ }^{26}$ E. H. Cirlin, H. M. Gebhard, and M. Shen, J. Macromol. Sci. Chem. A5, 981 (1971).

THE JOURNAL OF CHEMICAL PHYSICS

\title{
Angular Relaxation of the Symmetrical Top. II. The Rough Sphere*
}

\author{
K. L. Rider $\dagger$ AND M. Fixman \\ Chemistry Department, Yale University, New Haven, Connecticut 06520
}

(Received 1 May 1972)

\begin{abstract}
We investigate the reorientation of molecules in a bath of atoms without internal degrees of freedom. A Markoffian kinetic equation for the rough sphere model is formulated. A collision operator is derived and the appropriate kinetic equation for the two time orientational autocorrelation functions is solved for a variety of molecular parameters. It is found that the finite collision rough sphere model allows the retainment of greater dynamic coherence than does the Langevin model of the spherical top.
\end{abstract}

\section{INTRODUCTION}

We are concerned, in this paper, with the orientational properties of molecules in a simple fluid bath at equilibrium. The classical approach to molecular orientation is the diffusion equation used by Debye. ${ }^{1}$ If a molecule undergoes random walk in orientational configuration space, the equation describing its motion is the diffusion equation in orientational coordinates. The Legendre functions are eigenfunctions of this equation and the orientational correlation functions ${ }^{2}$ to all orders are exponential.

It is possible to extend the Debye model by randomizing the length of time of a step and allowing the molecules to have an angular velocity which is in some manner randomized at the end of each step. ${ }^{3}$ It is found that the process of randomization of the direction of angular velocity at the end of each step gives a good fit to the experimental data. However, complete randomization is not a consequence of any known intermolecular potential, realistic or otherwise.

The Langevin relaxation model, discussed by Fixman and Rider $^{2}$ (hereafter called I), yields solutions for the correlation function which involve a parameter that can be calculated microscopically. This model is in a sense a limit of the velocity independent collision model to be discussed. ${ }^{4}$ The limit is taken as collision frequency becomes large, the reduced mass of the collision constellation becomes small and the rate of momentum transfer remains the same. This implies many small transfers of angular momentum per unit time.

This paper will consider a model for orientational relaxation which takes into account large discontinuous changes in angular momentum. The system will be a dilute classical isotropic solution of rotating rough sphere molecules in an equilibrium bath and the energy coupling mechanism to be considered will be instantaneous hard core collisions. The treatment will complement that given in 1 .

A kinetic equation for the singlet distribution of an ensemble of molecules interacting with a bath of atoms but not interacting with each other will be sufficient to describe the complete behavior of a solution of molecules in the limit of infinite dilution. In considering bath-molecule interactions, it will be necessary to consider only those features of the interaction which 